\title{
Does Psychotherapy Change the Brain Functioning?
}

\author{
Myrthala Juárez-Treviño* \\ Department of Psychiatry, Hospital Universitario, México
}

*Corresponding author: Myrthala Juárez-Treviño, Department of Psychiatry, Hospital Universitario, Nuevo León, México.

To Cite This Article: Myrthala Juárez-Treviño. Does Psychotherapy Change the Brain Functioning?. Am J Biomed Sci \& Res. 2019 - 3(3). AJBSR. MS.ID.000670. DOI: 10.34297/AJBSR.2019.03.000670

Received: May 29, 2019 | Published: June 10, 2019

\begin{abstract}
In the past century psychoanalysis, psychological psychotherapy and biological psychiatry developed knowledge separately one from each other. In the last 20 years, neuroimaging has been determinate in displaying the effects of psychotherapy and pharmacotherapy in the brain functioning. Relevant neurophysiological findings in psychotherapeutic processes and neuroanatomy of the mental apparatus from a neuropsychoanalytical perspective are revised.
\end{abstract}

Keywords: Neuropsychoanalysis; Psychoanalysis; Psychotherapy; Brain function; Therapeutic change

Abbrevations: BT: Behavioral Therapy; CBF: Cerebral Blood Flow; CBT: Cognitive Behavioral Therapy; DLPFC: Dorsolateral Prefrontal Cortex; FMRI: Functional Magnetic Resonance Imaging; OCD: Obsessive Compulsive Disorder; PFC: Pre-Frontal Cortex; SPECT: Single Photon Emission Computed Tomography.

\section{Introduction}

Psychoanalysis and diverse types of psychotherapies have proved their benefits in treating different kinds of mental distress. But, what happens into the brain $\neg$ ? Can it be seen objectively? Psychological psychotherapy and biological psychiatry approaches regarding a patient's treatment are no longer seen as opposites, but complementarians. It is interesting to know how concepts like Freudian "repression", "transference", "free associate" have been identified into the central nervous system. Neuroimaging nowadays has made possible the Freud's dream of watching the neurons work. Psychoanalytical technique differs from other types of psychotherapy. The effects of psychoanalysis depend on the psychodynamic process of the resulting therapeutic relationship between the personality, empathy capacity, psychotherapeutic identity and attachment style of the analyst and the personality, psychodynamic structure, behavior and attachment style of the patient [1].

That is the reason why controlled experiments have been so hard to do in psychoanalysis besides the duration of a complete process. Replicability is a full challenge. Psychodynamic therapy embraces many of these factors. Other types of psychotherapy just focus on given tasks. This paper is about relevant notes to take in account at dawn of this branch of biomedical research.

\section{Psychotherapeutic Approaches}

\section{Behavioral therapy (BT) and Cognitive behavioral therapy (CBT)}

Roffman et al. [2] at the Massachusetts General Hospital and Linden at the University of Wales [2,3], published review articles about neuroimaging changes due to behavioral therapy (BT) and cognitive behavioral therapy (CBT) applied to obsessive compulsive disorder (OCD), phobias and depression. Their conclusions are alike, as follows:

1. Provocation of OCD symptoms has been associated with increases in cerebral blood flow (CBF) in the orbitofrontal cortex, anterior cingulate cortex, striatum and thalamus. After 10 weeks of BT, it was observed an uncoupling of hyperactivity in the right caudate, orbitofrontal cortex and thalamus (same as 10 weeks of treatment with fluoxetine) with decreased metabolism in the right caudate nucleus, a finding consistent with the well-established pathophysiology of the disorder.

2. Phobic patients show a prominent activation in limbic structures, including amygdala (the "fear center"), hippocampus and adjacent temporal cortex. After 8 weeks of CBT, there was a 
significant reduction of activity in these limbic and paralimbic regions; also showed decreased $\mathrm{CBF}$ in the periaqueductal gray area, which has been associated with defense behaviors.

3. Other studies are consistent with the model of BTrelated desensitization where modulation of limbic activation may involve either reductions of CBF in limbic and adjoining paralimbic regions or enhancement of $\mathrm{CBF}$ in ventral prefrontal cortex (PFC) or both when treating phobic patients.

4. Major depressive disorder has been associated with alterations in prefrontal brain activity with dorsal areas exhibiting decreased activity and ventral frontal regions demonstrating increased activity. Surprisingly in the CBT group, metabolism in multiple frontal regions including the dorso-lateral PFC decreased after therapy. This finding is contrary to expectation. The authors related the reduction in prefrontal metabolism to treatment-related diminution of active rethinking and reappraisal of emotional ideas. Patients who received paroxetine showed increased PFC metabolism. Since efficacy was comparable to CBT, it may imply that medication and CBT work through different mechanisms.

\section{Psychodynamic Psychotherapy}

Beutel et al. [4] investigated 14 panic patients before and after psychodynamic inpatient treatment. Based on the fMRI findings, at the beginning they showed stronger limbic activations (amygdala) to panic-specific words (e.g., dying, heart attack, collapse, etc.) compared to neutral or positive words. Intensive psychodynamic psychotherapy was conducted in an inpatient setting for 4 weeks. At the end, patients' state anxiety scores were no different from the normal controls and differences regarding brain-activation patterns were no longer discernable.

Beutel also cites the work of Viinamäki, Kuikka, and Tiihonen who studied by single photon emission computed tomography (SPECT) a couple of patients with borderline personality disorder who showed a reduced uptake of serotonin in the prefrontal cortex and the thalamus. One of them underwent psychotherapy for one year. After that, serotonin uptake was restored. Gerber and his colleagues [5] observed the role of the dorsolateral prefrontal cortex (DLPFC) in free association and concluded that "cognitive" concepts such as executive function, attention, and working memory and "analytic" concepts such as free association, defense and resistance all belong in a single category of mental functions. Understanding free association through its effect on executive function and the DLPFC, therapeutic action of psychodynamic cognitive and interpersonal treatments may not be fundamentally different.

\section{Neuropsychoanalysis}

Id, Ego, Superego: In short terms, the Id is the affective/ instinctual limbic system of the brain; the Ego is the inhibitor/ cognitive cortex; and the Superego is the frontal lobes. It is easy to contemplate "short-cuts" between the anatomical structures or misfunctioning to expect altered behavior.

There are, then, basic emotions, pulsions, instincts, wishes. Seven are the innate-predictive emotions:

\section{Seeking/Enthusiasm \\ 2. Lust \\ 3. Rage/Anger \\ 4. Care/Nurturance \\ 5. Play/Joy \\ 6. Fear/Anxiety \\ 7. Panic/Grief [6]}

All of them are needed for learning from good and bad experiences. Cerebral cortex records that experiences, so it inhibits impulses, plans behavior, thinks and imagines modulating inner emotions when required. Humans develop avoidant forms of thinking or feeling distress: they repress. Internal anxiety causes suffering that is avoided or distorted by defenses: some mature (repressive), some immature (pre-repressive) [7]. The Ego begins at the periphery of the body, with the sensory end-organs that convey coded information derived from the outside world to the cortex. The information is analyzed within the grey matter of the spinal cord, the cranial nerve nuclei in the brainstem and specific parts of the thalamus on its way to the unimodal cortical zones (the $\omega$ system of the Freud's "Project"). Heteromodal cortical zones are conceived as being the beginning of the mnemic systems of the Ego (the $\varphi$ systems). Mnemic systems occupy the posterior cortico-thalamic convexity and register and re-transcribe external perceptual information in "directories" linked to patterns. Significant patterns are encoded at deeper levels. This process is known as the structuralization of the Ego. In the right hemisphere, the structuralizations in heteromodal cortico-thalamic zones correspond to whole-object representations. In the left hemisphere, same structuralizations are associated with abstract quasi-spatial presentations of an audioverbal type described by Freud and Luria as symbolization [8].

Psychoanalytical process: Patients most seek psychotherapy because of distress. In psychoanalysis conscious symptoms are assumed to be the result of mechanisms of defense and formations of compromise which deal with multiple pre-conscious or unconscious factors.

Boeker et al. [1] point out that two are the basic psychoanalytic approaches to enable a therapeutic process:

1. To generate a patient's insight of the pre-conscious or unconscious aspects and parts of him- or herself.

2. The therapeutic relationship promotes an identification based upon introjection (of a good object) and empathy by constructing a new affective organization.

Interpretation is related to explicit memory (as part of the cortex). Implicit memory can be seen through repetitive transference. These implicit memories can be emptied of their quotas of affect through transference when a new affective configuration is established. Introjection may be the neurochemical basis of psychic change, since new neural circuits develop. Affective involvement is needed in neural plasticity that evolves after mechanisms of learning and their repetition. 
When a therapist 'takes a history', episodic memories from the patient emerge in a focused way. When the same patient evokes memories through free association, episodic recall occurs in a less organized, more random way. Random memories may engage wide networks of association cortex in frontal, parietal and temporal regions while focused memories activate selective verbal areas, including the Broca's one [2].

\section{Conclusion}

Despite the few studies to date, neuroimaging has revealed psychotherapy effects in the functional structure of the brain. So far, it is behavioral treatments that have been studied, with a brief duration. The longest study reported is one with a borderline patient after one year of treatment showing evident cerebral changes [9]. It is expected to find identifiable changes in larger processes. Systematic and well-controlled brain imaging studies are the future in neuroscience. Contributions between psychodynamic/ psychoanalytical psychotherapies and brain imaging may lead to more accurate diagnosis and therapeutic predictive markers determining the best approach to be prescribed. Boeker et al. [1] have told us about variables to think about for studying the way therapeutic processes change the stirring mind [1]. Challenge is at the door. There is a lot to come.

\section{Conflict of Interest}

None.

\section{References}

1. Boeker H, Richter A, Himmighoffen H, Ernst J, Bohleber L, et al. (2013) Essentials of psychoanalytic process and change: how can we investigate the neural effects of psychodynamic psychotherapy in individualized neuro-imaging? Front Hum Neurosci 7: 355.

2. Roffman JL, Marci CD, Glick DM, Dougherty DD, Rauch SL (2005) Neuroimaging and the functional neuroanatomy of psychotherapy. Psychol Med 35(10): 1385-1398.

3. Linden DEJ (2006) How psychotherapy changes the brain - the contribution of functional neuroimaging. Mol Psychiatry 11(6): 528538.

4. Beutel ME, Huber M (2008) Functional neuroimaging - can it contribute to our understanding of precesses of change? Neuropsychoanalysis 10(1): 5-16.

5. Gerber AJ (2009) Free Association as a Bridging Concept between Dynamic and Cognitive Processes and the Nature of Psychotherapeutic Change. Neuropsychoanalysis 11(2): 168-171.

6. Davis KL, Panksepp J (2018) The Emotional Foundations of Personality. A neurobiological and Evolutionary Approach. WW Norton \& Company, Inc. New York, USA.

7. Zellner M (2018) Repression and Defense. An overview of psychodynamic defenses, México.

8. Kaplan-Solms K, Solms M (2000) Clinical Studies in NeuroPsychoanalysis. Introduction to a Depth Neuropsychology. International Universities Press, Inc. Connecticut, USA.

9. Viinamäki H, Kuikka J, Tiihonen J, Johannes Lehtonen (1998) Change in monoamine transporter density related to clinical recovery: A casecontrol study. Nordic Journal of Psychiatry 52(1): 39-44. 